\title{
Analytical computational models for buckling and postbuckling of thin- walled stiffened composite panels
}

\author{
Jakob C. Schilling*, Christian Mittelstedt ${ }^{\dagger}$ \\ * Institute for Lightweight Construction and Design \\ Technical University of Darmstadt, Department of Mechanical Engineering \\ Otto-Berndt-Str. 2, 64287 Darmstadt, Germany \\ e-mail: jakob.schilling@klub.tu-darmstadt.de, web page: https://www.klub.tu-darmstadt.de/ \\ ${ }^{\dagger}$ Institute for Lightweight Construction and Design \\ Technical University of Darmstadt, Department of Mechanical Engineering \\ Otto-Berndt-Str. 2, 64287 Darmstadt, Germany
}

\begin{abstract}
The loss of stability is critical in regard to thin-walled composite structures which are widespread in weight critical applications. These applications include for example spacecraft, aircraft and marine vessels. For the example of omega-stringer stiffened panels, the buckling and postbuckling behavior is investigated using closed-form analytical approximate solutions. They are derived based on energy methods and their focus lies on local stability behavior. Hereby, different load cases are included and the quality of the computational models is assessed in comparison to Levy solutions or finite element analyses. The main benefits of the new models are in its reduced computational effort compared to numerical methods. This is especially relevant in preliminary design stages, where a large number of panel configurations have to be taken into account. Thus, the new analytical computational models aide to increase performance of thin walled composite structures in practical applications.
\end{abstract}

